\title{
Multistream Sensor Fusion-Based Prognostics Model for Systems with Single Failure Modes
}

\author{
Xiaolei Fang, Kamran Paynabar, Nagi Gebraeel \\ Georgia Institute of Technology
}

\begin{abstract}
Advances in sensor technology have facilitated the capability of monitoring the degradation of complex engineering systems through the analysis of multistream degradation signals. However, the varying levels of correlation with physical degradation process for different sensors, high-dimensionality of the degradation signals and cross-correlation among different signal streams pose significant challenges in monitoring and prognostics of such systems. To address the foregoing challenges, we develop a three-step multi-sensor prognostic methodology that utilizes multistream signals to predict residual useful lifetimes of partially degraded systems. We first identify the informative sensors via the penalized (log)location-scale regression. Then, we fuse the degradation signals of the informative sensors using multivariate functional principal component analysis, which is capable of modeling the cross-correlation of signal streams. Finally, the third step focuses on utilizing the fused signal features for prognostics via adaptive penalized (log)-location-scale regression. We validate our multi-sensor prognostic methodology using simulation study as well as a case study of aircraft turbofan engines available from NASA repository.
\end{abstract}

Keywords: Degradation modeling, functional variables selection, (log)-location-scale regression, multivariate functional principal components analysis, signal fusion 


\section{Introduction}

This paper focuses on developing a prognostic methodology for engineering systems being monitored by multiple sensors. Multiple sensors are used to capture different aspects of the failure process. Raw signals from these sensors are often transformed into degradation signals that can be used to predict residual useful lifetime (RUL). Most of the existing literature focuses either on modeling an individual degradation signal or on combining all the degradation signals together through some sort of fusion mechanism. There are various types of prognostic models that focus on single sensor applications. Examples include using random coefficients models [9, 49], Brownian motion process [31, 47, 48], Gamma process $[20,51,50]$, and Markov chains $[1,13]$. The second category are the models focusing on multi-sensor settings. They typically rely on combining all the available sensor signals using different types of fusion methods, such as neural networks [45], Hidden Markov models [41, 44], neuro-fuzzy systems [39, 37, 38, 40, 42], multilayer perceptron networks[43], health index models [46, 16, 52, 53] and functional principal components analysis [8].

In many multi-sensor applications, there exists some level of redundancy among the sensors. That is, more than one sensor may capture the same physical effects to a similar degree. In some other instances, signals from some sensors may have little or no relation to the underlying physics, thus compromising the accuracy of predicting failures. As a result, selecting the appropriate sensors before the fusion process may possibly lead to better failure predictability.

This paper builds on the existing body of literature by proposing a multi-sensor prognostic methodology that incorporates a systematic sensor selection procedure. Very few existing models formally incorporate a sensor selection procedure $[46,16]$. Nonetheless, the procedures are based on visual inspection or other subjective procedures, and thus may vary from one user to another. Our methodology consists of three steps shown in Figure 1. The first step is a formalized sensor selection algorithm that systematically identifies the most informative sensors that should be used to predict failure and RUL. This step is based upon a penalized variable selection methodology [34, 21]. The goal is to identify 


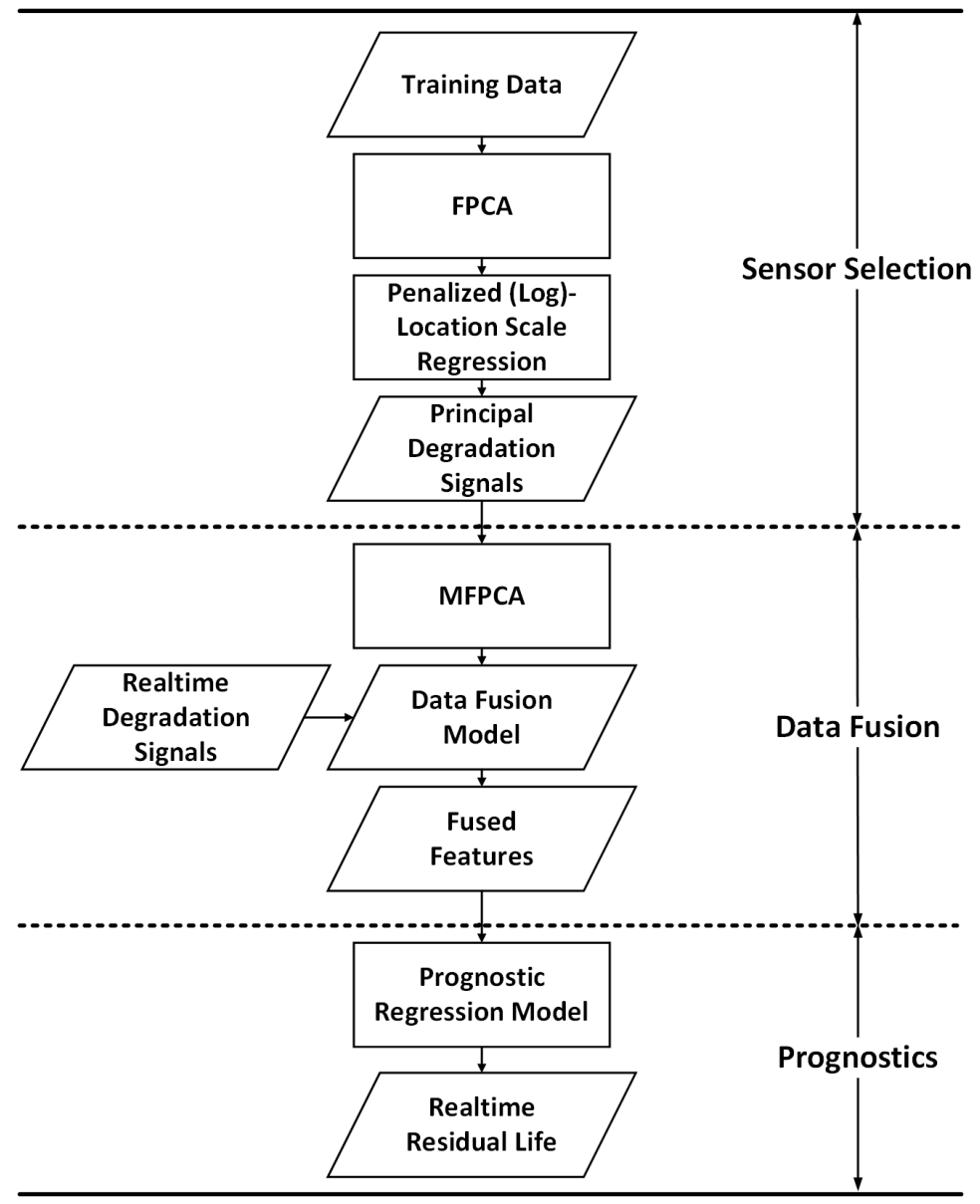

Figure 1: Multi-sensor fusion-based prognostics framework.

highly informative sensors that when combined together provide a relatively comprehensive yet precise characterization of the underlying physical degradation. The penalized variable selection is developed by combining functional principal components analysis (FPCA) with a penalized (log)-location-scale functional regression model. FPCA is a popular functional data analysis (FDA) technique that identifies the important sources of patterns and variations among functional data [23]. The degradation signals from each sensor are projected to a low-dimensional feature space spanned by the eigen-functions of the signals' covariance function and provides fused features called FPC-scores. The FPC-scores are then regressed against the time-to-failure (TTF) to identify the most informative sensors using a penalized (log)-location-scale regression model. (Log)-location-scale regression has been widely used 
in reliability engineering [17] and can be used with a variety of TTF distributions, such as (log)-normal, (log)-logistics, smallest extreme value, Weibull, etc.

The second step focuses on intelligently combining the degradation signals associated with the informative sensors identified by the sensor selection process. This is achieved by developing a signal fusion algorithm based on multivariate functional principal components analysis (MFPCA) [23]. MFPCA focuses on capturing the joint variation of multistream functional data. It works by applying ordinary FPCA on the concatenated degradation signals from different sensors. The benefit of using MFPCA is that it reduces dimensionality of the data and provides fused signal features in the form of MFPC-scores.

Finally, the third step focuses on utilizing the fused signal features for prognostics. This will be accomplished by using an adaptive penalized (log)-location-scale regression model, which estimates RUL and provides the means to continuously update these estimates as real-time signals are observed from fielded systems.

The remainder of the paper is organized as follows. In Section 2 we present the degradation modeling and sensor selection methodology used for identifying the most informative sensors whose signals will be used for prognostics. We then discuss the multistream data fusion algorithm in Section 3. Section 4 discusses the development of the prognostic model used for estimating and updating RULs of fielded engineering systems. The accuracy of the prognostic model is evaluated using a simulation study in Section 5 and aircraft turbofan engine degradation data from a physics-based simulation model developed by NASA in Section 6. Finally, the conclusion and future research directions are presented in Section 7.

\section{Signal model and sensor selection}

This framework focuses on systems whose failure is dominated by a single degradation process, which is being monitored by multiple sensors. We assume that raw signals from each individual sensor can be easily synthesized into degradation-based signals. Thus, each sensor has a corresponding degradation signal. Furthermore, we assume that there exists a 
historical database of degradation signals, a training dataset, that can be leveraged in model estimation. Ideally this database will contain high quality degradation signals from a set of (identical) systems along with their corresponding times-to-failure (TTF). The underlying premise of our multi-sensor prognostic methodology is that it is possible to identify a select subset of sensors that provides similar (and sometimes better) characterization of the degradation process, rather than relying on all the sensors used in monitoring. The benefits of doing this include a possible improvement in the accuracy of failure predictability as well as potential reduction in the costs of data acquisition and processing.

A variable selection methodology is utilized to develop our sensor selection procedure. At a high level, this is achieved by combining penalized (log)-location-scale regression with functional principal components analysis (FPCA) to identify the sensors the that are most correlated with the underlying degradation process and ultimately the system's TTF. We consider a training dataset of degradation signals for $N$ systems where each system is monitored by $P$ sensors. Let $s_{p}(t)$ for $p=1,2, \ldots, P$ denote the observed degradation signal of sensor $p$, such that $s_{p}(t)$ are independent noisy realizations of a smooth random function $x_{p}(\cdot)$ in a bounded time domain $[0, T]$ with unknown mean function $\mathbb{E}\left[x_{p}(t)\right]=\mu_{p}(t)$ and covariance function $C_{p}\left(t, t^{\prime}\right)=\operatorname{Cov}\left(x_{p}(t), x_{p}\left(t^{\prime}\right)\right)$. We express the degradation signal from sensor $p$ of system $i$ as follows:

$$
s_{i, p}(t)=x_{i, p}(t)+\epsilon_{i, p}(t) ; \quad i=1,2, \ldots, N,
$$

where $\epsilon_{i, p}(t)$ are assumed to be independent and identically distributed (i.e., i.i.d.) errors with mean zero and variance $\sigma_{p}^{2}$, and $x_{i, p}(t)$ and $\epsilon_{i, p}(t)$ are independent.

Using Mercer's theorem [12], the covariance matrix $C_{p}\left(t, t^{\prime}\right)$ can be expanded as follows;

$$
C_{p}\left(t, t^{\prime}\right)=\sum_{k=1}^{\infty} \lambda_{k, p} \phi_{k, p}(t) \phi_{k, p}\left(t^{\prime}\right), t, t^{\prime} \in[0, T]
$$

where $\phi_{k, p}(t)$ for $k=1,2, \ldots$ are the orthogonal eigen-functions and $\lambda_{1, p} \geq \lambda_{2, p} \geq \ldots$ are the ordered nonnegative eigen-values. Using these eigen-functions, $s_{i, p}(t)$ can be expressed 
as linear combinations of the orthogonal basis functions as follows:

$$
s_{i, p}(t)=\mu_{p}(t)+\sum_{k=1}^{\infty} \xi_{i, k, p} \phi_{k, p}(t)+\epsilon_{i, p}(t),
$$

where $\xi_{i, k, p}$ for $k=1,2, \ldots$, are known as FPC-scores, which are uncorrelated random variables with mean zero and variance $\mathbb{E}\left(\xi_{i, k, p}^{2}\right)=\lambda_{k, p}$. Generally, the eigenvalues $\lambda_{k, p}$ for $k=1,2, \ldots$, decrease rapidly and only a small number of eigenvalues would suffice to capture most information of multistream signals and the rest are approximately zero. Therefore, it is often sufficient to use only the eigenfunctions corresponding to significantly nonzero eigenvalues to accurately approximate the signals. Consequently, $s_{i, p}(t)$ can be approximated as shown below;

$$
s_{i, p}(t)=\mu_{p}(t)+\sum_{k=1}^{K_{p}} \xi_{i, k, p} \phi_{k, p}(t)+\epsilon_{i, p}(t) .
$$

where $K_{p}$ is the number of significantly nonzero eigenvalues. The value of $K_{p}$ can usually be chosen by using cross validation (CV) and the Akaike information criterion (AIC) [7]. In practice, the fraction of variance explained $(\mathrm{FVE})$ is another efficient way to determine $K_{p}$ (see [8] for more details).

\subsection{Sensor selection methodology}

Based on the premise that the TTF of a system can be predicted by its degradation signals, we establish the following (log)-location-scale regression model for modeling the TTF (i.e., $f_{i}$ ) as a function of the degradation signals (i.e., $\left.\left\{s_{i, p}(t)\right\}_{p=1}^{P}\right)$ :

$$
\operatorname{Pr}\left(y_{i} \leq y\right)=\Omega\left(\frac{y-\pi\left(s_{i, p}(t)\right)}{\sigma}\right),
$$

where $y_{i}=f_{i}$ if $f_{i}$ follows a location-scale distribution and $y_{i}=\log \left(f_{i}\right)$ if $f_{i}$ follows a log-location-scale distribution. $\Omega$ is the cumulative distribution function (i.e., cdf) of the

location-scale distribution, $\sigma$ is the scale parameter, and $\pi\left(s_{i, p}(t)\right)$ is the location parameter, 
which is a function of some explanatory variables. Here we assume that $\pi\left(s_{i, p}(t)\right)$ is a function of degradation signals, i.e., $\pi\left(s_{i, p}(t)\right)=\alpha_{0}+\sum_{p=1}^{P} \int_{0}^{T} \alpha_{p}(t) s_{i, p}(t) d t$, where $\alpha_{0}$ is the intercept, $\alpha_{p}(t)$ is the coefficient function.

The (log)-location-scale regression model in Equation (5) can be solved using maximum likelihood estimation (MLE), which yeilds the following optimization criterion:

$$
\max _{\alpha_{0}, \alpha_{p}(t), \sigma}\left\{-N \log (\sigma)+\sum_{i=1}^{N} \omega\left(\frac{y_{i}-\alpha_{0}-\sum_{p=1}^{P} \int_{0}^{T} \alpha_{p}(t) s_{i, p}(t) d t}{\sigma}\right)\right\},
$$

where $\omega(\cdot)$ is the probability density function (i.e., pdf) of the location-scale distribution. For example, $\omega(x)=1 / \sqrt{2 \pi} \exp \left(-x^{2} / 2\right)$ for normal distribution, $\omega(x)=\exp (x-\exp (x))$ for smallest extreme value distribution and $\omega(x)=\exp (x) /(1+\exp (x))^{2}$ for logistic distribution. Criterion (6) is nonconvex, which causes computational problems. To address this challenge, we apply the following re-parameterization: $\tilde{\sigma}=1 / \sigma, \widetilde{y}_{i}=y_{i} / \sigma, \widetilde{\alpha}_{0}=\alpha_{0} / \sigma$ and $\widetilde{\alpha}_{p}(t)=$ $\alpha_{p}(t) / \sigma$. As a result, criterion (6) turns into the following criterion:

$$
\max _{\widetilde{\alpha}_{0}, \widetilde{\alpha}_{p}(t), \widetilde{\sigma}}\left\{N \log (\widetilde{\sigma})+\sum_{i=1}^{N} \omega\left(\widetilde{y}_{i}-\widetilde{\alpha}_{0}-\sum_{p=1}^{P} \int_{0}^{T} \widetilde{\alpha}_{p}(t) s_{i, p}(t) d t\right)\right\},
$$

Sensor selection is performed by incorporating a variable selection procedure into the MLE criterion expressed in (7). Classical variable selection techniques like stepwise regression are often computationally intensive and sometimes unstable. On the other hand, regularization-based techniques like non-negative garrote (NNG) [2], LASSO [27], SCAD [5], elastic net [35], and adaptive LASSO [36] exhibit some advantages with respect to selection stability and prediction accuracy. In this work, we use group non-negative garrote (GNNG) penalty [33] to penalize the group of coefficients corresponding to each degradation signal, i.e., $\left\{\alpha_{p}(t)\right\}$. Consider the following parameterization, $\widetilde{\alpha}_{p}(t)=\widehat{\alpha}_{p}(t) d_{p}$, where $\widehat{\alpha}_{p}(t)$ is a known weight function and $d_{p} \geq 0$ is the shrinking factor for signal $p$, then based on criterion (7), the penalized maximum log-likelihood function using the GNNG can be expressed 
as follows;

$$
\max _{\widetilde{\alpha}_{0}, d_{p}, \widetilde{\sigma}}\left\{N \log (\widetilde{\sigma})+\sum_{i=1}^{N} \omega\left(\widetilde{y}_{i}-\widetilde{\alpha}_{0}-\sum_{p=1}^{P} \int_{0}^{T} \widehat{\alpha}_{p}(t) d_{p} s_{i, p}(t) d t\right)-\lambda \sum_{p=1}^{P} d_{p}\right\} \text {, s.t. } d_{p} \geq 0
$$

where $\lambda$ is the tuning parameter and $d_{p}$ represents the importance of the group (or sensor) $p$.

Selecting the most informative sensors is accomplished by optimizing criterion (8). Hereafter, the degradation signals associated with the selected sensors will be referred to as the principle degradation signals (PD-signals). Any sensor whose corresponding $d_{p}$ is non-zero is considered informative (important), and its corresponding degradation signal is designated as one of the PD-signals that will be used for prognostics, estimating the RUL of the system. Recall that the eigen-functions of the covariance functions of $\left\{x_{i, p}(t)\right\}_{i=1}^{N}$ form a complete orthogonal basis, which means that the functions $\widehat{\alpha}_{p}(t)$ can be expanded as $\widehat{\alpha}_{p}(t)=\sum_{k=1}^{\infty} \beta_{k, p} \phi_{k, p}(t)$, where $\beta_{k, p}$ is the coefficient. Similarly, since the eigen-values $\lambda_{k, p}$ for $k=1,2, \ldots$ decrease rapidly (as mentioned earlier), the expansion can be truncated by using the first $K_{p}$ terms. Thus, we get $\widehat{\alpha}_{p}(t)=\sum_{k=1}^{K_{p}} \beta_{k, p} \phi_{k, p}(t)$. Substituting $s_{i, p}(t)$ and $\widehat{\alpha}_{p}(t)$ into the location parameter in criterion (8) yields the following expression (the details of the derivation are given in Appendix A):

$$
\pi\left(s_{i, p}(t)\right)=\widetilde{\alpha}_{0}+\sum_{p=1}^{P} \int_{0}^{T} \widehat{\alpha}_{p}(t) d_{p} s_{i, p}(t) d t=\beta_{0}+\sum_{p=1}^{P} d_{p} \sum_{k=1}^{K_{p}} \beta_{k, p} \xi_{i, k, p}
$$

where $\xi_{i, k, p}$ is the FPC-score defined earlier in Equation (4). Consequently, The penalized maximum likelihood function criterion expressed in (8) can be rewritten in the following 
form;

$$
\max _{\beta_{0}, d_{p}, \widetilde{\sigma}}\left\{N \log (\widetilde{\sigma})+\sum_{i=1}^{N} \omega\left(\widetilde{y}_{i}-\beta_{0}-\sum_{p=1}^{P} d_{p} \sum_{k=1}^{K_{p}} \beta_{k, p} \xi_{i, k, p}\right)\right\}-\lambda \sum_{p=1}^{P} K_{p} d_{p} \text {, s.t. } d_{p} \geq 0
$$

where $\beta_{k, p}$ is the vector of the weights associated with sensor $p$. Generally, we can use maximum likelihood, lasso, or ridge estimates of $\beta_{k, p}$ as the weights [34]. As mentioned earlier, the sensors with $\widehat{d}_{p}>0$ are selected, and their corresponding signals are designated as the PD-Signals.

\subsection{Model estimation}

The optimization model expressed in Equation (10) can be solved using the coordinate descent algorithm used in [3]. However, this requires first estimating the FPC-scores, $\xi_{i, k, p}$, which can be achieved using the training dataset. To do this, we first estimate the mean $\mu_{p}(t)$ and covariance functions $C_{p}\left(t, t^{\prime}\right)$ for $p=1,2, \ldots, P$. To simplify notation, and without loss of generality, we assume that the sampling frequency for all sensors is the same.

Local linear regression is used to estimate $\mu_{p}(t)[4,14]$. The estimated mean function for signal $p$, which we denote as $\widehat{\mu}_{p}(t)$, can be obtained by minimizing the following loss function,

$$
\min _{a_{p}, b_{p}} \sum_{i=1}^{N} \sum_{j=1}^{J_{i}} W\left(\frac{t_{j}-t}{w_{p}}\right)\left\{s_{i, p}\left(t_{j}\right)-a_{p}-b_{p}\left(t-t_{j}\right)\right\}^{2}
$$

where $\left\{t_{j}\right\} \in[0, T]$ for $j=1, \ldots, J_{i}$, denotes discrete observation time points, and $J_{i}$ represents the length of degradation signals in system $i . w_{p}$ is the smoothing bandwidth, which is selected by using the one-curve-leave-out cross-validation method presented in [22], and $W(\cdot)$ is a Gaussian kernel function. The estimated mean function is $\widehat{\mu}_{p}(t)=\widehat{a}_{p}$, where $\widehat{a}_{p}$ is the solution to Equation (11).

The covariance function, $C_{p}\left(t, t^{\prime}\right)$, is also estimated using local linear regression[4, 14]. From Equation (1), we can see that $\operatorname{Cov}\left(s_{p}\left(t_{j}\right), s_{p}\left(t_{k}\right)\right)=\operatorname{Cov}\left(x_{p}\left(t_{j}\right), x_{p}\left(t_{k}\right)\right)+\sigma^{2} \delta_{j, k}$, where 
$\delta_{j, k}=1$ if $t_{j}=t_{k}$ and 0 otherwise. Let $G_{i, p}\left(t_{j}, t_{k}\right)=\left(s_{i, p}\left(t_{j}\right)-\widehat{\mu}_{p}\left(t_{j}\right)\right)\left(s_{i, p}\left(t_{k}\right)-\widehat{\mu}_{p}\left(t_{k}\right)\right)$ be the "raw" covariances of degradation signal $p$ of system $i$, we have $E\left[G_{i, p}\left(t_{j}, t_{k}\right)\right]=$ $\operatorname{Cov}\left(x_{p}\left(t_{j}\right), x_{p}\left(t_{k}\right)\right)+\sigma^{2} \delta_{j, k}$. In other words, the noise term only lies on the diagonal elements of the raw covariances. Therefore, the diagonal elements of $G_{i, p}\left(t_{j}, t_{k}\right)$ are removed and only off-diagonal elements of the raw covariances are considered for estimation [32]. The covariance function is estimated by minimizing the following loss function;

$$
\min _{a_{p}^{\prime}, b_{p}^{\prime}, c_{p}^{\prime}} \sum_{i=1}^{N} \sum_{1 \leq j \neq k \leq J_{i}} W\left(\frac{t_{j}-t}{w_{p}^{\prime}}, \frac{t_{k}-t^{\prime}}{w_{p}^{\prime}}\right)\left\{G_{i, p}\left(t_{j}, t_{k}\right)-a_{p}^{\prime}-b_{p}^{\prime}\left(t-t_{j}\right)-c_{p}^{\prime}\left(t^{\prime}-t_{k}\right)\right\}^{2},
$$

where $w_{p}^{\prime}$ is the smoothing bandwidth, and $W(\cdot)$ is a bivariate Gaussian kernel function. The estimated covariance function is obtained as $\widehat{C}_{p}\left(t, t^{\prime}\right)=\widehat{a}_{p}^{\prime}$, for $t, t^{\prime} \in[0, T]$ where $\widehat{a}_{p}^{\prime}$ is the solution to Equation (12).

Once the covariance function has been estimated, our next step is to estimate its eigenfunctions $\phi_{k, p}(t)$ and eigen-values $\lambda_{k, p}$. This is achieved by solving the following eigenequations:

$$
\int_{0}^{T} \widehat{C}_{p}\left(t, t^{\prime}\right) \widehat{\phi}_{k, p}(t) d t=\widehat{\lambda}_{k, p} \widehat{\phi}_{k, p}\left(t^{\prime}\right)
$$

where $\int_{0}^{T} \widehat{\phi}_{k, p}(t) \widehat{\phi}_{m, p}(t) d t=1$ if $k=m$ and 0 otherwise.

Equation (13) can be solved by discretizing the estimated covariance surface $\widehat{C}_{p}\left(t, t^{\prime}\right)[22]$. The FPC-scores are estimated using $\widehat{\xi}_{i, k, p}=\int_{0}^{T}\left(s_{i, p}(t)-\widehat{\mu}_{p}(t)\right) \widehat{\phi}_{k, p}(t) d t$, which is approximated numerically by $\widehat{\xi}_{i, k, p}=\sum_{j=0}^{J_{i}}\left(\left(s_{i, p}\left(t_{j}\right)-\widehat{\mu}_{p}\left(t_{j}\right)\right) \widehat{\phi}_{k, p}\left(t_{j}\right)\left(t_{j}-t_{j-1}\right)\right) ; t_{0}=0$. Finally, the estimated $\widehat{\xi}_{i, k, p}$ can then be substituted in the penalized maximum likelihood criterion defined in Equation (10) to estimate the importance of each sensor, i.e., $d_{p}$. This allows us to identify the most informative sensors. Recall that the degradation signals associated with these select sensors are referred to as the PD-signals. These form the basis for predicting the RULs of partially degraded systems. 


\section{Multistream signal fusion model}

In this section, we focus on how to efficiently combine the PD-signals such that their crosscorrelations can be leveraged to provide accurate predictions of RUL. To do this, we develop a multistream signal fusion methodology based on multivariate functional principal components analysis (MFPCA). MFPCA is an extension of the FPCA framework. Whereas FPCA focuses on identifying the important sources of variation among a single type of functional data, MFPCA focuses on capturing the joint variation of multistream functional data. One of the key benefits of using MFPCA is that it provides a way to characterize the cross-correlation between the PD-signals measured by different sensors. By modeling the relationship between different informative sensors, we can better characterize the underlying degradation process, and therefore improve prediction accuracy. Another advantage of using MFPCA is that it reduces the dimensionality of the PD-signals, and provides fused features (in the form of MFPC-scores) that become the predictors in our subsequent prognostic degradation modeling framework.

\subsection{Signal fusion using MFPCA}

Based on [23], MFPCA works by first concatenating different sources of functional data into a single vector. Next, FPCA is applied to the concatenated vector in a conventional manner. Note that signal fusion is only applied to the PD-signals associated with the sensors identified by the sensor selection methodology. We begin by assuming that the PD-signals of unit $i$ are independent noisy realizations of an $M$-dimensional stochastic process $\boldsymbol{x}(\cdot)$ in a bounded time domain $[0, T]$ with unknown mean function $\boldsymbol{\mu}(t)$ and covariance function $\boldsymbol{C}\left(t, t^{\prime}\right)$, where $M$ is the number of selected sensors. Here, $\boldsymbol{\mu}(t)$ represents the combined underlying deterministic trend of the degradation process, and $\boldsymbol{C}\left(t, t^{\prime}\right)$ represents the deviation from the underlying degradation trend due to system-to-system degradation variability. 
Therefore, the PD-signals for system $i$ can be expressed as follows:

$$
\boldsymbol{s}_{i}(t)=\boldsymbol{x}_{i}(t)+\boldsymbol{\epsilon}_{i}(t)
$$

for $i=1, \ldots, N$, where $N$ is the system number; $\boldsymbol{s}_{i}(t)=\left(s_{i, 1}(t), \ldots, s_{i, M}(t)\right)^{\top}, \boldsymbol{x}_{i}(t)=$ $\left(x_{i, 1}(t), \ldots, x_{i, M}(t)\right)^{\top}$ and $\boldsymbol{\epsilon}_{i}(t)=\left(\epsilon_{i, 1}(t), \ldots, \epsilon_{i, M}(t)\right)^{\top} ; \boldsymbol{x}_{i}(t)$ and $\boldsymbol{\epsilon}_{i}(t)$ are assumed to be independent. Note that the covariance function $\boldsymbol{C}\left(t, t^{\prime}\right)=\mathbb{E}\left[(\boldsymbol{x}(t)-\boldsymbol{\mu}(t))\left(\boldsymbol{x}\left(t^{\prime}\right)-\boldsymbol{\mu}\left(t^{\prime}\right)\right)^{\top}\right], \quad t, t^{\prime} \in$ $[0, T]$ is an $M \times M$ block matrix with the following form;

$$
\boldsymbol{C}\left(t, t^{\prime}\right)=\left(\begin{array}{ccc}
C_{1,1}\left(t, t^{\prime}\right) & \ldots & C_{1, M}\left(t, t^{\prime}\right) \\
\vdots & \ddots & \vdots \\
C_{M, 1}\left(t, t^{\prime}\right) & \ldots & C_{M, M}\left(t, t^{\prime}\right)
\end{array}\right)
$$

with $C_{g, h}\left(t, t^{\prime}\right)=\operatorname{Cov}\left(x_{g}(t), x_{h}\left(t^{\prime}\right)\right)$, for $g=1, \ldots, M$ and $h=1, \ldots, M$.

Mercer's theorem [12] is also used to decompose the covariance function $\boldsymbol{C}\left(t, t^{\prime}\right)$ as follows;

$$
\boldsymbol{C}\left(t, t^{\prime}\right)=\sum_{k=1}^{\infty} \eta_{k} \boldsymbol{\psi}_{k}(t) \boldsymbol{\psi}_{k}\left(t^{\prime}\right)^{\top}
$$

where $\boldsymbol{\psi}_{k}(t)=\left(\psi_{k, 1}(t), \ldots, \psi_{k, M}(t)\right)^{\top}$ for $k=1,2, \ldots$, are the eigenfunctions and $\eta_{1} \geq \eta_{2} \geq$ $\ldots$, are the ordered nonnegative eigenvalues. Hence, $\boldsymbol{x}_{i}(t)$, can be rewritten as,

$$
\boldsymbol{x}_{i}(t)=\boldsymbol{\mu}(t)+\sum_{k=1}^{\infty} \zeta_{i, k} \boldsymbol{\psi}_{k}(t)
$$

where $\zeta_{i, k}=\int_{0}^{T}\left(\boldsymbol{x}_{i}(t)-\boldsymbol{\mu}(t)\right)^{\top} \boldsymbol{\psi}_{k}(t) d t=\sum_{m=1}^{M} \int_{0}^{T}\left(x_{i, m}(t)-\mu_{m}(t)\right) \psi_{k, m}(t) d t$ for $k=1,2, \ldots$, are the MFPC-scores. These scores are independent random variables with mean $\mathbb{E}\left[\zeta_{i, k}\right]=0$ and variance $\mathbb{E}\left[\zeta_{i, k}^{2}\right]=\eta_{k}$. The signal model expressed in (14) can now be expressed as,

$$
\boldsymbol{s}_{i}(t)=\boldsymbol{\mu}(t)+\sum_{k=1}^{K} \zeta_{i, k} \boldsymbol{\psi}_{k}(t)+\boldsymbol{\epsilon}_{i}(t)
$$

where similar to the decomposition in Section 2, the model is truncated by choosing the first 
$K$ eigenvalues and $K$ is also chosen using CV, AIC or FVE criterion (see Equation (4)).

\subsection{Estimating the fused signal features}

Given a historical training dataset of signals, we can estimate the signal model expressed in Equation (18). Let $\left\{t_{j}\right\}$ for $j=1, \ldots, J$, where $t_{j} \in[0, T]$ denote discrete observation time points, where $J$ is the total number of observations for each system. We first estimate the mean function $\boldsymbol{\mu}(t)$, where $\boldsymbol{\mu}(t)=\left(\mu_{1}(t), \ldots, \mu_{M}(t)\right)^{\top}$. The $m$ th element of the mean function $\boldsymbol{\mu}(t)$ is estimated using $\widehat{\mu}_{m}\left(t_{j}\right)=\frac{1}{N} \sum_{i=1}^{N} s_{i, m}\left(t_{j}\right)$ for $m=1, \ldots, M$, where $N$ is the system number.

To estimate the covariance matrix, we note that $\boldsymbol{C}\left(t, t^{\prime}\right)$ is a $M \times M$ block matrix. Each block $C_{g, h}\left(t, t^{\prime}\right)$ is estimated individually using $\widehat{C}_{g, h}\left(t_{j}, t_{k}\right)=\frac{1}{N-1} \sum_{i=1}^{N}\left(s_{i, g}\left(t_{j}\right)-\widehat{\mu}_{g}\left(t_{j}\right)\right)\left(s_{i, h}\left(t_{k}\right)-\right.$ $\left.\widehat{\mu}_{h}\left(t_{k}\right)\right)$, for $g=1, \ldots, M, h=1, \ldots, M, j=1, \ldots, J$ and $k=1, \ldots, J$. Since $\boldsymbol{C}\left(t, t^{\prime}\right)$ is a symmetric matrix, we only need to estimate $C_{g, h}\left(t, t^{\prime}\right)$ with $g \geq h$.

Next, we estimate the MFPC-scores $\zeta_{i k}$. Recall that $\boldsymbol{C}\left(t, t^{\prime}\right)=\sum_{k=1}^{\infty} \eta_{k} \boldsymbol{\psi}_{k}(t) \boldsymbol{\psi}_{k}\left(t^{\prime}\right)^{\top}$, where the eigenfunctions $\boldsymbol{\psi}_{k}(t)$ and the eigenvalues $\eta_{k}$ can now be estimated by solving the following eigen equations:

$$
\int_{0}^{T} \widehat{\boldsymbol{C}}\left(t, t^{\prime}\right) \widehat{\boldsymbol{\psi}}_{k}(t) d t=\widehat{\eta}_{k} \widehat{\boldsymbol{\psi}}_{k}\left(t^{\prime}\right)
$$

where $\int_{0}^{T} \widehat{\boldsymbol{\psi}}_{k}(t) \widehat{\boldsymbol{\psi}}_{l}(t) d t=1$ if $l=k$ and 0 otherwise. Equation (19) is solved by discretizing the estimated covariance surface $\widehat{\boldsymbol{C}}\left(t, t^{\prime}\right)$ (details can be found in [22]). In other words, the MFPC-scores can be estimated using numerical integration where $\widehat{\zeta}_{i, k}=\sum_{m=1}^{M} \sum_{j=1}^{J_{i}}\left(s_{i, m}\left(t_{j, m}\right)-\right.$ $\left.\left.\widehat{\mu}_{m}\left(t_{j, m}\right)\right) \widehat{\phi}_{k, m}\left(t_{j, m}\right)\left(t_{j, m}-t_{j-1, m}\right)\right)$, and where $t_{0}=0$. Note that the MFPC-scores represent the collective (reduced) features of the PD-signals, which will be used to estimate the RUL of the system. 


\section{Residual useful lifetime prediction and real-time up- dating}

Predicting the RUL is accomplished by developing a (log)-location-scale regression model between the system TTFs and the PD-signals estimated earlier. To do this, we let $\boldsymbol{s}_{i}(t)$ denote a vector of PD-signals and $f_{i}$ the corresponding TTF of system $i$. The (log)-locationscale regression model relating these two factors is expressed below;

$$
\operatorname{Pr}\left(y_{i} \leq y\right)=\Omega\left(\frac{y-\rho_{0}-\int_{0}^{T} \boldsymbol{\rho}(t)^{\top} \boldsymbol{s}_{i}(t) d t}{\sigma}\right)
$$

where $y_{i}=f_{i}$ if $f_{i}$ follows a location-scale distribution and $y_{i}=\log \left(f_{i}\right)$ if $f_{i}$ follows a log-location-scale distribution; $\rho_{o}$ is the intercept; and $\boldsymbol{\rho}(t)=\left(\rho_{1}(t), \ldots, \rho_{M}(t)\right)^{\top}$ is the regression coefficient function. The coefficient funciton can be expanded using the eigenfunctions of $\left\{\boldsymbol{x}_{i}(t)\right\}_{i=1}^{N}$ as $\boldsymbol{\rho}(t)=\sum_{k=1}^{\infty} \theta_{k} \boldsymbol{\psi}_{k}(t)$. Similar to the derivation in Appendix A, the location parameter in Equation (20) can be expressed as $\boldsymbol{\pi}\left(\boldsymbol{s}_{i}(t)\right)=\rho_{0}+\int_{0}^{T} \boldsymbol{\rho}(t)^{\top} \boldsymbol{s}_{i}(t) d t=$

$\theta_{0}+\sum_{k=1}^{K} \theta_{k} \zeta_{i, k}$, where $\zeta_{i, k}$ are the MFPC-scores defined in Equation (17), and $\theta_{0}$ and $\theta_{k}$ are the coefficients. As a result, Equation (20) can be reexpressed as

$$
\operatorname{Pr}\left(y_{i} \leq y\right)=\Omega\left(\frac{y-\theta_{0}-\sum_{k=1}^{K} \theta_{k} \zeta_{i, k}}{\sigma}\right)
$$

The MLE is used to solve the regression model in Equation (21), which yields the following optimization criterion:

$$
\max _{\theta_{0}, \theta_{k}, \sigma}\left\{-N \log (\sigma)+\sum_{i=1}^{N} \omega\left(\frac{y_{i}-\theta_{0}-\sum_{k=1}^{K} \theta_{k} \zeta_{i, k}}{\sigma}\right)\right\},
$$

In order to transfer (22) to a convex optimization criterion, we apply the following re- 
parameterization: $\widetilde{\sigma}=1 / \sigma, \widetilde{y}_{i}=y_{i} / \sigma, \widetilde{\theta}_{0}=\theta_{0} / \sigma, \widetilde{\theta}_{k}=\theta_{k} / \sigma$ (see [8] for more details). In addtion, to improve the prediction accuracy, we use non-negative garrote [2] for penalization, which yields the following penalized maximum likelihood function criterion;

$$
\max _{\widetilde{\theta}_{0}, d_{k}, \widetilde{\sigma}}\left\{N \log (\widetilde{\sigma})+\sum_{i=1}^{N} \omega\left(\widetilde{y}_{i}-\widetilde{\theta}_{0}-\sum_{k=1}^{K} d_{k} \widehat{\theta}_{k} \zeta_{i, k}\right)\right\}-\lambda \sum_{p=1}^{K} d_{k} \text {, s.t. } d_{k} \geq 0
$$

where $\widehat{\theta}_{k}$ is the initial regression coefficients estimated using MLE without penalization, $d_{k}$ is the shrinkage factor, and $\lambda$ is the tuning parameter.

Our goal is to predict and update, in near real-time, the RUL of partially degraded systems that are still operating in the field. To do this, PD-signals observed from fielded systems (hereafter referred to as observed PD-Signals) are used to update the fused signals features, which are then used to update the predicted RUL. This is accomplished by combining the observed PD-signals from a specific (fielded) system with PD-signals of the training dataset, and using our signal fusion algorithm to compute updated MFPC-scores. The updated MFPC-scores are then used revise the predicted lifetime using the (log)-location-scale regression model estimated earlier in Equation (23). The RUL is obtained by subtracting the current observation time.

To calculate the scores, MFPCA requires that the PD-signals from each type of sensor share the same time domain. That is, when combining the PD-signals observed from the field with the PD-signals from the training dataset, signals for each sensor must share the same time domain. We note that the systems of the training sets have different TTFs. Thus, there are no guarantees that for a given sensor, the PD-signals from various systems will share the same time domain. To address this limitation, we leverage recent developments in functional regression. Specifically, we utilize the adaptive functional regression approach proposed by [18].

The basis of adaptive functional regression is that training systems whose lifetime is smaller than the current observation time of the PD-signal are removed from the training dataset. In other words, they are not used to recalculate the MFPC-scores. Only the PD- 
signals of the training systems whose lifetime is bigger than the current observation time are combined with the PD-signals observed from the fielded system to update the MFPC-scores. The chosen training PD-signals are then truncated at the current observation time. This way all the signals involved share the same time domain as the observed PD-signal. Figure 2 from [7] illustrates how the adaptive updating approach would apply to a single sensor scenario. In each graph, the dotted lines represent the entire set of training signals. The continuous part marks the part of the data that is used to estimate the MFPC-scores. The signal marked with the thick continuous line represents the portion of the degradation signal of the fielded system that has been observed up to time $t^{*}$.

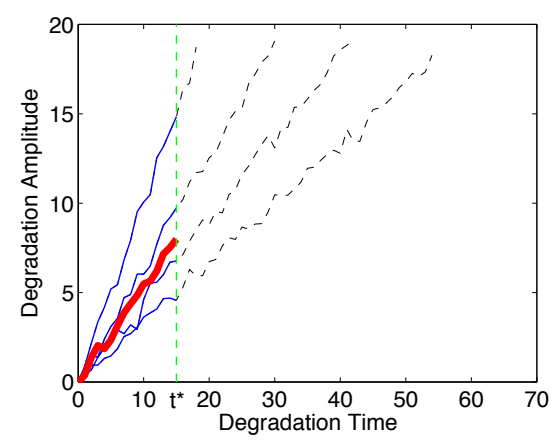

(a) Updating at time $t^{*}=15$

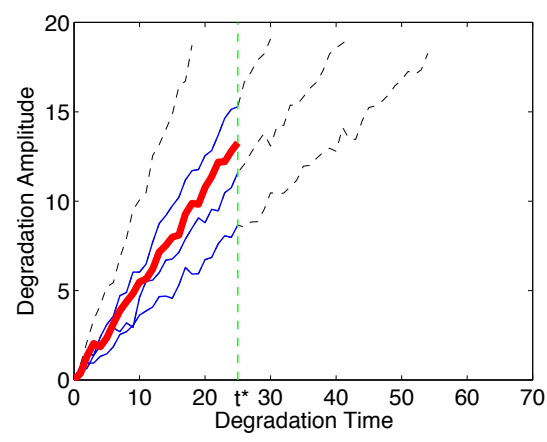

(b) Updating at time $t^{*}=25$

Figure 2: Method of updating the model as time advances [7]

We summarize our prognostic model as follows. Each time new PD-signals are observed from a fielded system, we select the training PD-signals that satisfy the criteria mentioned earlier and truncate them at the current observation time. Next, we apply MFPCA to the selected PD-signals (the ones selected from the training dataset along with the observed signal), and estimate revised MFPC-scores. A penalized regression model is then defined by setting the revised MFPC-scores as the dependent variables and setting the TTF corresponding to the selected training PD-signals as the dependent variables. Finally, the lifetime of the fielded system can be estimated.

The most time-consuming computation in our prognostic model is MFPCA, which consists of two key operations: covariance matrix estimation and eigen-decomposition. Suppose the system number is $N$, the selected sensor number is $M$ and the observation number for 
each sensor is $J$, the computational complexity of covariance matrix estimation and eigendecomposition are $\mathcal{O}\left(N M^{2} J^{2}\right)$ and $\mathcal{O}\left(M^{3} J^{3}\right)$, respectively. As a result, the complexity of MFPCA is $\mathcal{O}\left(N M^{2} J^{2}+M^{3} J^{3}\right)$, which is high when $M$ and $J$ are large. This in turn shows the importance of our sensor selection procedure, which often can tremendously reduce the number of sensors (reduce $M$ ) involved in the prognostics model. For the case where the number of selected sensor is large, say thousands, a computationally efficient solution can be found in [8].

\section{Simulation study}

In this section, we conduct a simulation study to validate the proposed prognostic methodology. We consider two (log)-location-scale regression distributions commonly used in reliability, the normal and lognormal distributions. For each distribution, we evaluate the performance of our approach in terms of the effectiveness of the sensor selection model and the accuracy of predicting the RUL. We compare the performance of our methodology, designated "selection," with the case that all sensors are used for predicting RUL, designated "no selection." In both approaches, the number of FPC-scores are chosen so that more than $95 \%$ of signal variations are explained by the chosen scores. The tuning parameters of Equations (8) and (23) are determined using the leave-one-out cross-validation method. Several values for the tuning parameters within an applicable range are used to train the model. Then, the validation error is calculated for different parameter values. Tuning parameter leading to the least mean square error is chosen to be the optimal tuning parameter.

\subsection{Simulation model}

We consider a system monitored by 40 sensors. We assume that only 4 sensors are informative. In other words, the system TTF can be accurately predicted using the degradation signals from these 4 sensors. We also assume that the set of non-informative sensors are divided into two groups. The first group consists of 16 sensors whose degradation signals have a relatively low correlation with the degradation process and therefore the TTF. The 
second group consists of 20 sensors whose signals are pure noise and exhibit no trends.

Two hundred instances of this system are generated. A randomly subset of 160 systems are chosen for training while the remaining 40 instances are used for testing. For each instance $i ; i=1, \ldots, 200$, we begin by simulating the underlying degradation path of the system using the following functional form; $s_{i}(t)=-\theta_{i} / \ln (t)$, where $\theta_{i} \sim N\left(1,0.25^{2}\right)$ and $0 \leqslant t<1$. The TTF is computed as the first time point that the underlying degradation trajectory, $s_{i}(t)$, crosses the threshold $D$, where $D=2$. Next, degradation signals from the 40 sensors are simulated as follows:

(a) Degradation signals from the informative sensors are generated using the following model $s_{i, p}(t)=-\theta_{i, p} / \ln (t)+\epsilon_{i, p}(t)$, where $p=1, \ldots, 4$ and $\epsilon_{i, p}(t) \sim N\left(0,0.1^{2}\right)$. Since informative sensors are highly correlated with the underlying degradation process, we generate $\theta_{i, p} ; p=1, \ldots, 4$ from the following conditional distribution $\theta_{i, p} \mid \theta_{i} \sim N\left(1,0.25^{2}\right)$ such that the correlation between $\theta_{i, p}$ and $\theta_{i}$ is a uniform random number chosen from the interval $[0.8,0.99]$.

(b) Degradation signals of the non-informative sensors in group 1 (i.e., $s_{i, p}(t) ; p=$ $5, \ldots, 20)$ are also simulated similarly. However, pairwise correlation is randomly chosen from the interval $[0.1,0.6]$.

(c) Degradation signals of the non-informative sensors in group 2 are simulated from $s_{i, p}(t)=\epsilon_{i, p}(t) ; p=21, \ldots, 40$ where $\epsilon_{i, p}(t) \sim N(0,1)$.

Samples of 200 simulated signals for each sensor type are shown in figure 3 . The whole simulation procedure is replicated 100 times.

\subsection{Results and analysis}

To test the effectiveness of the sensors selection methodology, we begin by applying the sensor selection model discussed in Section 3 to the simulated dataset.

To account for the variability in the length of the signals (discussed in Section 4), multiple training subsamples are generated based on the length of signals (or equivalent TTFs). We first, sort TTFs such that $T T F_{1} \leq T T F_{2} \leq \cdots \leq T T F_{S}$, where $S \leq 160$. Next, we define

subsample $j$ as the systems whose TTFs are greater than or equal to $T T F_{j}$, for $j=1, \ldots, S$. 


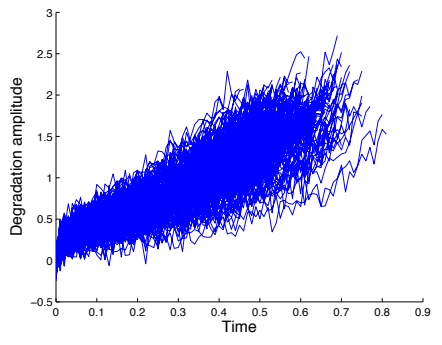

(a) Informative sensors

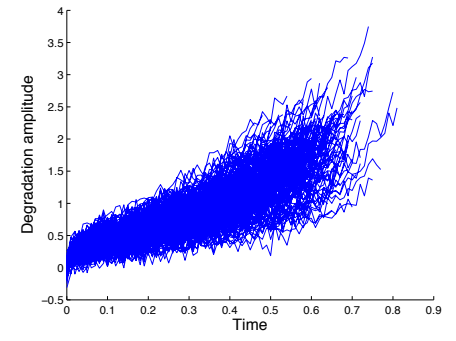

(b) non-informative sensors (poor correlation)

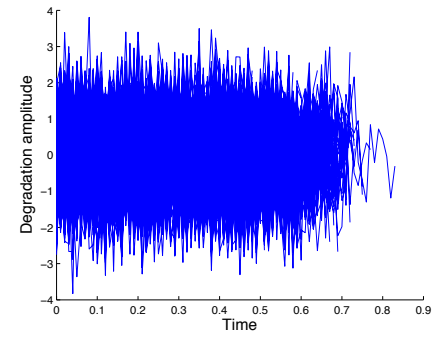

(c) non-informative sensors (pure noise)

Figure 3: Example signals of different type of sensors

As a case in point, subsample 1 includes all 160 training systems, subsample 2 also includes all training systems excluding the system with the smallest TTFs, and so forth.

The proposed sensor selection method is applied to all of these $S$ subsmaples and the weighted selection rate is computed by using the following equation;

$$
r_{p}=\frac{\sum_{j=1}^{S} I_{p} N_{j}}{\sum_{j=1}^{S} N_{j}} \times 100 \%,
$$

where $r_{p}$ is the selection rate for sensor $p, I_{p}=1$ if sensor $p$ is selected by subsample $j$ and 0 otherwise, and $N_{s}$ is the system size of subsample $j$. The selection results for normal and lognormal distributions are reported in Table 1 and Table 2, respectively.

Table 1: Sensor selection results for normal regression model.

\begin{tabular}{l|l|l}
\hline & Selected & Dropped \\
\hline Informative sensors & $94.6 \%$ & $5.4 \%$ \\
\hline Noninformative sensors & $2.8 \%$ & $97.2 \%$ \\
\hline
\end{tabular}

Table 2: Sensor selection results for lognormal regression model.

\begin{tabular}{l|l|l}
\hline & Selected & Dropped \\
\hline Informative sensors & $96.5 \%$ & $3.5 \%$ \\
\hline Noninformative sensors & $2.3 \%$ & $97.7 \%$ \\
\hline
\end{tabular}

As shown in the tables above, the lognormal and normal models selected $94.6 \%$ and $96.5 \%$ 


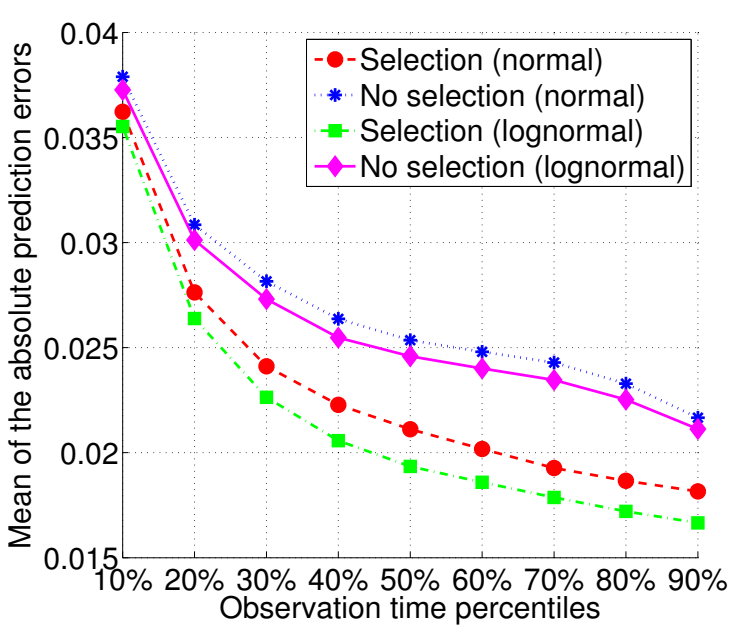

(a) Mean

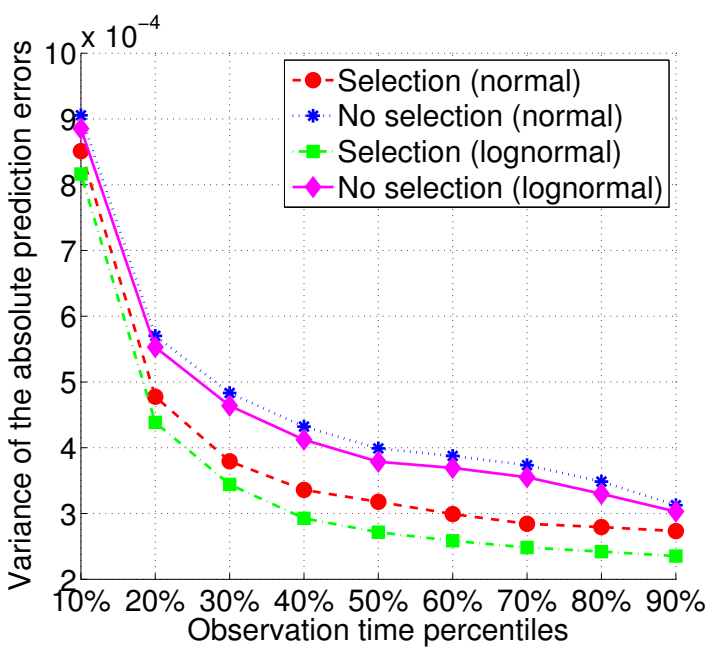

(b) Variance

Figure 4: Mean and variance of the absolute prediction errors.

of informative sensors, respectively. Both lognormal and normal models perform similarly with regards to removing non-informative sensors.

Next, we evaluate the accuracy of predicting the RUL of the simulated test systems using our proposed methodology for the normal and lognormal cases-designated "selection (normal)" and "selection (lognormal)"-and compare it with the benchmark model designated "no selection (normal)" and "no selection (lognormal)". For our methodology, the PD-signals identified by the sensor selection procedure are first combined using the signal fusion algorithms presented in Section 3. The fused features (MFPC-scores) are then used for prognostics. Signals from each test system are combined with the training dataset to predict and update its RUL using the time-varying functional regression framework discussed earlier in Section 4. Residual lifetimes are evaluated at the following life percentiles: $10 \%, 20 \%, \ldots, 90 \%$, where for example the $10^{\text {th }}$ life percentile implies that $10 \%$ of the system's lifetime was attained at the time the prediction was evaluated. The prediction errors are calculated using the following expression:

$$
\text { Absolute Prediction Error }=\frac{\mid \text { Estimated Life }- \text { Actual Life } \mid}{\text { Actual Life }} .
$$


Plot (a) of Figure 4 shows the mean of absolute prediction errors and Plot (b) shows the variance of the absolute prediction errors. The two plots confirm that our proposed sensor selection approach has significantly better prediction accuracy (mean) and precision (variance) for both the normal and lognormal scnearios. The improvement is more pronounced at higher life percentiles. For example, at the $70^{\text {th }}$ life percentile, the mean prediction errors for "selection (lognormal)" and "no selection (lognormal)" are 0.018 and 0.023 respectively, which indicates $20 \%$ improvement in the prediction accuracy achieved by the sensor selection methodology.

Figure 4 also indicated that the lognormal model outperforms the normal model. A possible explanation is that the degradation signals used in this study are generated from the following model $s_{i, p}(t)=-\theta_{i, p} / \ln (t)+\epsilon_{i, p}(t)$, in which the $\operatorname{logarithmic}$ TTF (i.e., $\log \left(f_{i}\right)$ ) can be shown to be a linear function of the MFPC-scores of degradation signals (i.e., $\zeta_{i, k}$ ). Therefore, the lognormal model is supposed to be more suitable than normal model to capture the regression relationship between TTF and MFPC-scores. In reality, we may use model selection criteria (e.g., AIC, Bayesian Information Criterion) to determine the proper choice of the (log)-location-scale distribution.

\section{Case study: aircraft turbofan engine application}

In this case study, multi-sensor degradation data from an aircraft turbofan engine is simulated using a physics-based simulator. The dataset, available from [25] is comprised of the following; (1) degradation signals from 100 training engines that were run to failure, (2) degradation signals from an additional 100 test engines whose operation was prematurely terminated at random time points prior to their failure time, and (3) the real TTFs of the 100 test engines. Each engine was monitored using 21 sensors, which are listed in Table 3. Since the degradation signals for some of the sensors $(1,5,6,10,16,18,19)$ show no trend, independent and identically distributed white noise are added to them. Figure 6 shows the degradation signals of the 21 sensors for the 100 training engines. 


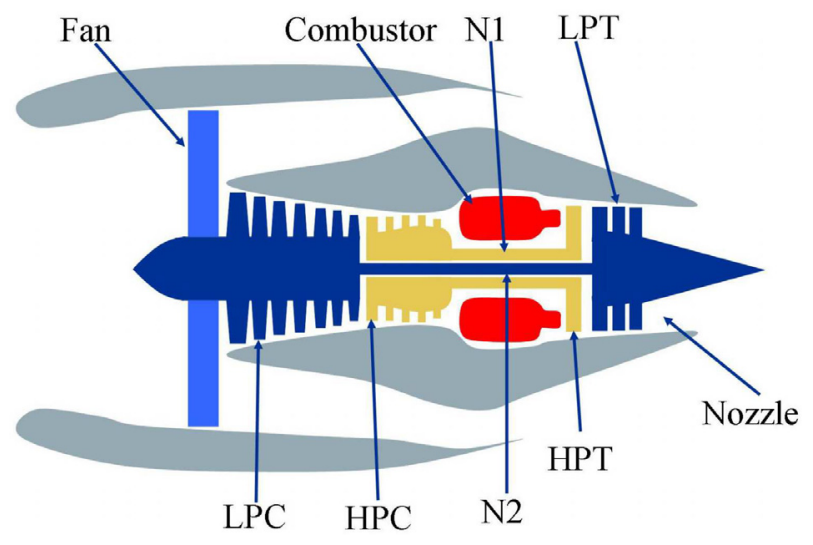

Figure 5: Simplified diagram of engine simulated in C-MAPSS [6].

The turbofan engine degradation dataset was simulated using commercial modular aeropropulsion system simulation (C-MAPSS), a simulation module developed in Matlab and Simulink environment. The C-MAPSS can be used for simulating a realistic commercial turbofan engine of 90,000 lb thrust class [6]. By setting the value of several input parameters and specifying the operation conditions, a user can simulate the effects of faults and deterioration in one or more of the rotating components (Fan, LPC, HPC, HPT and LPT) of engines. Figure 5 shows the main elements of the engine model used in C-MAPSS. The dataset used in this case study was simulated based on the assumption that the degradation of engines resulted from wear and tear of one single component (i.e., high pressure chamber, HPC) of the engines based on the usage pattern, under constant operating condition [24] Also, in this dataset, the damage accumulation during a particular flight cannot be calculated directly based on flight duration and flight conditions, and we have to rely on the degradation signals recorded by multiple sensors during or right after each flight.

We applied our prognostic methodology by first performing sensor selection using four different (log)-location-scale distributions, i.e., normal, lognormal, SEV and Weibull distributions. Similar to the procedure presented in the simulation study (Section 5), we created 65 subsamples with different time domains for sensor selection. The proposed sensor selection approach is then applied to these 65 subsamples and the selection rate of each sensors is computed and reported in Table 4. Sensors with the selection rate higher than $50 \%$ are 
Table 3: 21 outputs for degradation modeling

\begin{tabular}{llll}
\hline Index & Symbol & Description & units \\
\hline \hline 1 & T2 & Total temperature at fan inlet & ${ }^{\circ} \mathrm{R}$ \\
2 & T24 & Total temperature at LPC outlet & ${ }^{\circ} \mathrm{R}$ \\
3 & T30 & Total temperature at HPC outlet & ${ }^{\circ} \mathrm{R}$ \\
4 & T50 & Total temperature at LPT outlet & ${ }^{\circ} \mathrm{R}$ \\
5 & P2 & Pressure at fan inlet & psia \\
6 & P15 & Total pressure in bypass-duct & psia \\
7 & P30 & Total pressure at HPC outlet & psia \\
8 & Nf & Physical fan speed & rpm \\
9 & Nc & Physical core speed & rpm \\
10 & epr & Engine pressure ratio (P50/P2) & - \\
11 & Ps30 & Static pressure at HPC outlet & $\mathrm{psia}$ \\
12 & phi & Ratio of fuel flow to Ps30 & $\mathrm{pps} / \mathrm{psi}$ \\
13 & NRf & Corrected fan speed & $\mathrm{rpm}$ \\
14 & NRc & Corrected core speed & $\mathrm{rpm}$ \\
15 & BPR & Bypass Ratio & - \\
16 & farB & Burner fuel-air ratio & - \\
17 & htBleed & Bleed Enthalpy & - \\
18 & Nf_dmd & Demanded fan speed & $\mathrm{rpm}$ \\
19 & PCNfR_dmd & Demanded corrected fan speed & $\mathrm{rpm}$ \\
20 & W31 & HPT coolant bleed & $\mathrm{lbm} / \mathrm{s}$ \\
21 & W32 & LPT coolant bleed & $\mathrm{lbm} / \mathrm{s}$ \\
\hline
\end{tabular}

selected as informative sensors. As can be seen from Table 4, sensors 4, 15, 17, 20, were selected by normal and lognormal distributions and sensor 4, 17, 20 were selected by SEV and Weibull distribution.

To select the most suitable model, we calculate the corresponding AIC for these distributions and select the one with the lowest AIC. The calculated AIC values for normal, lognormal, SEV and Weibull are 989.9, 977.7, 1027.2 and 1006.8, respectively, suggesting that the lognormal distribution is likely the most appropriate. Subsequently, the PD-signals of the selected sensors are then fused using MFPCA and the fused features are used to develop the subsequent prognostic model based on lognormal regression.

The performance of our model is compared with two benchmarks in terms of prediction accuracy and precision. The first benchmark, "no selection", is the same benchmark used in 

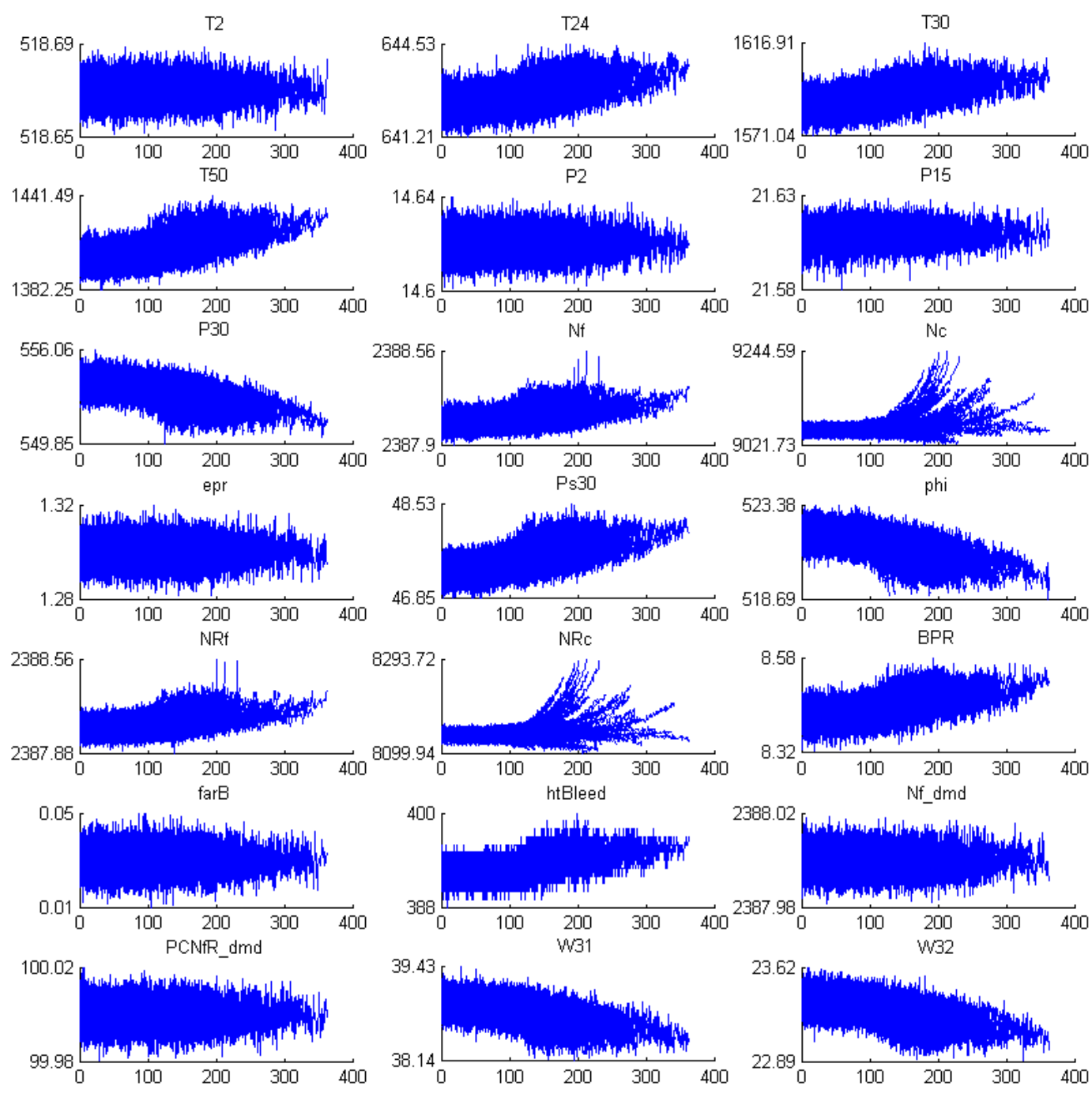

Time (Cycles)

Figure 6: Degradation signals from 21 sensors of 100 training aircraft turbofan engines.

the simulation study in which all the sensors were used for prediction. The second benchmark is the health index methodology ("HI+selection") proposed by [16] in which the authors used the same aircraft dataset. In [16], the authors visually selected 11 sensors out of the total 21 sensors, and created a composite degradation index by fusing these 11 individual sensor signals. Since all degradation signals include a stationary phase with no degradation at the 
Table 4: Sensor selection results for aircraft engine dataset.

\begin{tabular}{l|l|l|l|l}
\hline Sensor & Normal & Lognormal & SEV & Weibull \\
\hline 1 & $0 \%$ & $0 \%$ & $0 \%$ & $0 \%$ \\
\hline 2 & $35.0 \%$ & $45.0 \%$ & $15.0 \%$ & $30.0 \%$ \\
\hline 3 & $22.5 \%$ & $30.0 \%$ & $17.5 \%$ & $22.5 \%$ \\
\hline 4 & $\mathbf{5 5 . 0 \%}$ & $\mathbf{5 5 . 0 \%}$ & $\mathbf{6 2 . 5 \%}$ & $\mathbf{6 5 . 0 \%}$ \\
\hline 5 & $0 \%$ & $0 \%$ & $0 \%$ & $0 \%$ \\
\hline 6 & $0 \%$ & $0 \%$ & $0 \%$ & $0 \%$ \\
\hline 7 & $12.5 \%$ & $7.5 \%$ & $17.5 \%$ & $15.0 \%$ \\
\hline 8 & $0 \%$ & $0 \%$ & $0 \%$ & $0 \%$ \\
\hline 9 & $0 \%$ & $0 \%$ & $0 \%$ & $0 \%$ \\
\hline 10 & $0 \%$ & $0 \%$ & $0 \%$ & $0 \%$ \\
\hline 11 & $35.0 \%$ & $20.0 \%$ & $45.0 \%$ & $45.0 \%$ \\
\hline 12 & $0 \%$ & $0 \%$ & $0 \%$ & $0 \%$ \\
\hline 13 & $0 \%$ & $0 \%$ & $0 \%$ & $0 \%$ \\
\hline 14 & $0 \%$ & $0 \%$ & $0 \%$ & $0 \%$ \\
\hline 15 & $\mathbf{6 7 . 5} \%$ & $\mathbf{7 0 . 0} \%$ & $27.5 \%$ & $42.5 \%$ \\
\hline 16 & $0 \%$ & $0 \%$ & $0 \%$ & $0 \%$ \\
\hline 18 & $\mathbf{8 2 . 5} \%$ & $\mathbf{8 5 . 0} \%$ & $\mathbf{5 5 . 0 \%}$ & $\mathbf{6 5 . 0 \%}$ \\
\hline 19 & $0 \%$ & $0 \%$ & $0 \%$ & $0 \%$ \\
\hline 20 & $0 \%$ & $0 \%$ & $0 \%$ & $0 \%$ \\
\hline 21 & $\mathbf{7 7 . 5} \%$ & $\mathbf{9 0 . 0} \%$ & $\mathbf{5 5 . 0 \%}$ & $\mathbf{6 7 . 5 \%}$ \\
\hline
\end{tabular}

beginning period [24], we calculate the prediction errors at only the $50 \%, 60 \%, \ldots, 90 \%$ of lifetime in a similar manner to the simulation study.

The mean and variance of the absolute prediction errors are summarized in Figure 7. Comparing the mean and variance of the absolute prediction errors of our method with those of benchmarks indicates the overall superiority of our model and as expected the importance of the sensor selection. The mean prediction errors of "selection" is lower than those of two other benchmarks at all life percentiles. In addition, our method has signifficantly smaller variance than the health index method, especially at earlier life percentiles, which again supports the benefits of sensor selection. 


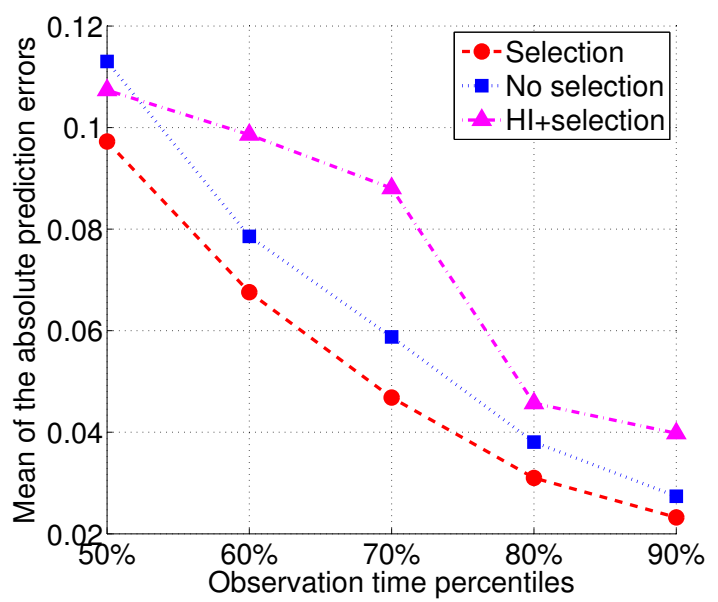

(a) Mean

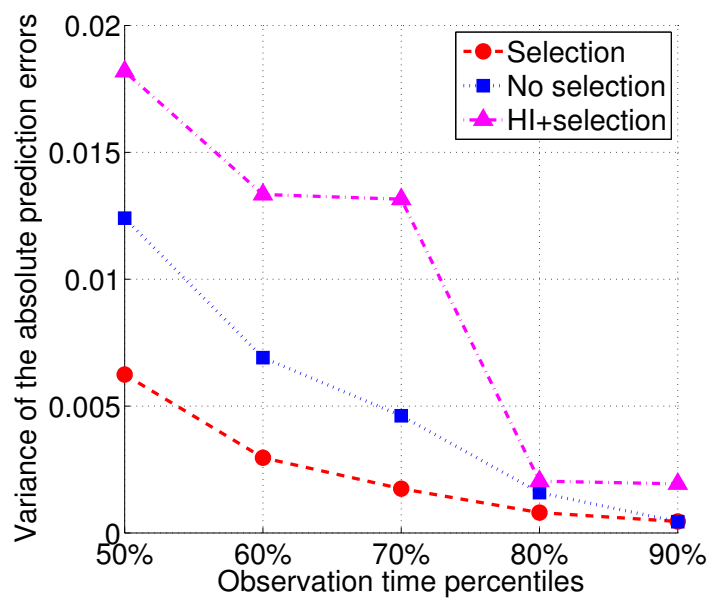

(b) Variance

Figure 7: Residual life prediction errors for multi-sensor aircraft turbofan engines.

\section{Conclusion}

Prognostic of complex systems monitored by multiple sensors is an important yet challenging problem. In this paper, we proposed a multi-sensor prognostic methodology that utilizes multistream signals to predict RULs of partially degraded systems. The proposed methodology consists of three steps. First, a sensor selection procedure was developed based on penalized (log)-location-scale functional regression. Specifically, we used the FPCA to extract the features of degradation signals, and then applied penalized regression to select informative sensors. In the second step, we utilized the MFPCA technique to fuse the PD-signals while considering the inter-relationship of signal streams. Finally, using the extracted fused features from MFPCA, an adaptive (log)-location-scale regression model with regularization was built for dynamic prediction of RULs.

We studied and compared the performance of our proposed methodology with different benchmarks using simulations. The results indicated that our methodology outperforms the benchmarks in terms of both the mean and variance of prediction errors. Moreover, simulation results showed that the proposed sensor selection procedure can effectively remove non-informative sensors, which in turn resulted in a more accurate and precise predictions. We also validated the effectiveness of our methodology using a simulated aircraft turbofan 
engine dataset from NASA repository. The results indicated that the sensor selection algorithm in our model can improve both the prediction accuracy and precision. The model developed in this paper only focuses on the multi-sensor systems with single failure mode. Development of a multistream prognostic methodology for systems with multiple failure modes is an important topic for future research. 


\section{Appendix A}

The location parameter in criterion (8) is expressed as follows:

$$
\pi\left(s_{i, p}(t)\right)=\widetilde{\alpha}_{0}+\sum_{p=1}^{P} \int_{0}^{T} \widetilde{\alpha}_{p}(t) s_{i, p}(t) d t
$$

Recall that $\widetilde{\alpha}_{p}(t)=\widehat{\alpha}_{p}(t) d_{p}(t), \widehat{\alpha}_{p}(t)=\sum_{k=1}^{\infty} \beta_{k, p} \phi_{k, p}(t)$ and $s_{i, p}(t)=\mu_{p}(t)+\sum_{k=1}^{\infty} \xi_{i, k, p} \phi_{k, p}(t)+$ $\epsilon_{i, p}(t)$, we have

$$
\begin{aligned}
\pi\left(s_{i, p}(t)\right) & =\widetilde{\alpha}_{0}+\sum_{p=1}^{P} \int_{0}^{T} \widetilde{\alpha}_{p}(t)\left\{\mu_{p}(t)+\sum_{k=1}^{\infty} \xi_{i, k, p} \phi_{k, p}(t)+\epsilon_{i, p}(t)\right\} \\
& =\widetilde{\alpha}_{0}+\sum_{p=1}^{P} \int_{0}^{T} \widetilde{\alpha}_{p}(t) \mu_{p}(t) d t+\sum_{p=1}^{P} \int_{0}^{T}\left\{\widehat{\alpha}_{p}(t) d_{p}\left(\sum_{k=1}^{\infty} \xi_{i, k, p} \phi_{k, p}(t)\right)\right\} d t+\sum_{p=1}^{P} \int_{0}^{T} \widetilde{\alpha}_{p}(t) \epsilon_{i, p}(t) d t \\
& =\beta_{0}+\sum_{p=1}^{P} \int_{0}^{T}\left\{d_{p} \sum_{k=1}^{\infty} \beta_{k, p} \phi_{k, p}(t)\right\}\left\{\sum_{k=1}^{\infty} \xi_{i, k, p} \phi_{k, p}(t)\right\} d t+\varepsilon_{i} \\
& =\beta_{0}+\sum_{p=1}^{P} d_{p} \sum_{k=1}^{\infty} \beta_{k, p} \xi_{i, k, p}+\varepsilon_{i} \\
& =\beta_{0}+\sum_{p=1}^{P} d_{p} \sum_{k=1}^{K_{p}} \beta_{k, p} \xi_{i, k, p}
\end{aligned}
$$

where $\beta_{0}=\widetilde{\alpha}_{0}+\sum_{p=1}^{P} \int_{0}^{T} \widetilde{\alpha}_{p}(t) \mu_{p}(t) d t$ is the intercept. 


\section{References}

[1] Bogdanoff J. L. and Kozin F. (1985), Probabilistic Models of Cumulative Damage, John Wiley \& Sons, New York.

[2] Breiman, L. (1995). "Better subset regression using the nonnegative garrote," Technometrics, 37(4), 373-384.

[3] Friedman, J., Hastie, T., \& Tibshirani, R. (2010), "Regularization paths for generalized linear models via coordinate descent," Journal of statistical software, 33(1), 1.

[4] Fan, J., and Gijbels, I. (1996), Local Polynomial Modelling and Its Applications, London: Chapmanand Hall.

[5] Fan, J., and Li, R. (2001). "Variable selection via nonconcave penalized likelihood and its oracle properties," Journal of the American Statistical Association, 96(456), 13481360.

[6] Frederick, D., DeCastro, J., and Litt, J. (2007), "User's Guide for the Commercial Modular Aero-Propulsion System Simulation (CMAPSS)," NASA/ARL, Technical Manual TM2007-215026.

[7] Fang X., Zhou R., and Gebraeel N. (2015), "An adaptive functional regression-based prognostic model for applications with missing data," Reliability Engineering 83 System Safety, 133, 266-274.

[8] Fang X., Paynabar K., and Gebraeel N. (2015), "Scalable Prognostic Models for Largescale Condition Monitoring Applications," IIE Transactions, Under review.

[9] Gebraeel, N. Z., Lawley, M. A., Li, R., and Ryan, J. K. (2005), "Residual-life distributions from component degradation signals: a Bayesian approach," IIE Transactions, 37(6), 543-557. 
[10] Hall, D. L., Llinas, J. (2001), Handbook of Multisensor Data Fusion, CRC Press, Boca Raton, FL.

[11] Jardine, A. K., Lin, D., and Banjevic, D. (2006), "A review on machinery diagnostics and prognostics implementing condition-based maintenance," Mechanical systems and signal processing, 20(7), 1483-1510.

[12] Karhunen, K. (1947), "Uber lineare Methoden in derWahrscheinlichkeitsrechnung", $A n$ nales Academiae Sciientiarum Fennicae, Series AI: Mathematica-Physica, 37, 3-79.

[13] Kharoufeh J. and Cox S. (2005), "Stochastic models for degradation based reliability", IIE Transactions, 37, 533-542.

[14] Loader, C. (1999), Local Regression and Likelihood (Vol. 47), New York: Springer.

[15] Lawless, J., and Crowder, M. (2004), "Covariates and random effects in a gamma process model with application to degradation and failure," Lifetime Data Analysis, $10(3), 213-227$.

[16] Liu K., Gebraeel N., and Shi J., (2013). "A Data-Level Fusion Model for Developing Composite Health Indices for Degradation Modeling and Prognostic Analysis," IEEE Transactions on Automation Science and Engineering, 10(3), 652-664.

[17] Meeker, W. Q. (1991), "Accelerated Testing: Statistical Models, Test Plans, and Data Analyses," Technometrics, 33(2), 236-238.

[18] Müller, H.G. and Zhang, Y. (2005), "Time-varying functional regression for predicting remaining lifetime distributions from longitudinal trajectories," Biometrics, 61, 10641075.

[19] Nelson, W. (1990), Accelerated life testing: statistical models, data analysis and test plans, Wiley, New York. 
[20] Park C. and Padgett W. (2005), "New cumulative damage models for failure using stochastic processes as initial damage," IEEE Transactions on Reliability, 54, 530-540.

[21] Paynabar, K., Jin, J., and Reed, M. (2013), "Informative Sensor and Feature Selection via Hierarchical Non-Negative Garrote," Under review.

[22] Rice, J., and Silverman, B. (1991), "Estimating the Mean and Covariance Structure Nonparametrically When the Data are Curves," Journal of the Royal Statistical Society Ser. B, 53, 233-243.

[23] Ramsay, J. O., Silverman, B. W (2005), Functional data analysis, Wiley Online Library.

[24] Saxena, A., Goebel, K., Simon, D., Eklund, N. (2008b), "Damage propagation modeling for aircraft engine run-to-failure simulation," In Prognostics and Health Management, 2008. PHM 2008. International Conference on.

[25] Saxena, A. and Goebel, K. (2008), "C-MAPSS Data Set," NASA Ames Prognostics Data Repository, [http://ti.arc.nasa.gov/project/prognostic-data-repository], NASA Ames, Moffett Field, CA.

[26] Simon, N., and Tibshirani, R. (2012), "Standardization and the group lasso penalty," Statistica Sinica, 22(3), 983.

[27] Tibshirani, R. (1996), "Regression shrinkage and selection via the lasso," Journal of the Royal Statistical Society. Series B (Methodological), 267-288.

[28] Tseng, S., Balakrishnan, N. and Tsai, C. (2009), "Optimal step-stress accelerated degradation test plan for gamma degradation processes," IEEE Transactions on Reliability, $58,611-618$.

[29] Whitmore, G. A. and Schenkelberg, F. (1997), "Modelling accelerated degradation data using Wiener diffusion with a time scale transformation," Lifetime Data Analysis, 3(1),27-45. 
[30] Wang, L., Chen, G., Li, H. (2007), "Group SCAD regression analysis for microarray time course gene expression data," Bioinformatics, 23(12), 1486-1494.

[31] Wang, X., and Xu, D. (2010), "An inverse Gaussian process model for degradation data," Technometrics, 52(2), 188-197.

[32] Yao, F., Müller, H. G., Wang, J. L. (2005), "Functional data analysis for sparse longitudinal data," Journal of the American Statistical Association, 100(470), 577-590.

[33] Yuan, M., and Lin, Y. (2006), "Model selection and estimation in regression with grouped variables," Journal of the Royal Statistical Society: Series B (Statistical Methodology), 68(1), 49-67.

[34] Yuan, M., and Lin, Y. (2007), "On the non-negative garrotte estimator," Journal of the Royal Statistical Society: Series B (Statistical Methodology), 69(2), 143-161.

[35] Zou, H., and Hastie, T. (2005), "Regularization and variable selection via the elastic net," Journal of the Royal Statistical Society: Series B (Statistical Methodology), 67(2), 301-320.

[36] Zou, H. (2006), "The adaptive lasso and its oracle properties," Journal of the American statistical association, 101(476), 1418-1429.

[37] Ramasso, E., \& Gouriveau, R. (2010, January). Prognostics in switching systems: Evidential Markovian classification of real-time neuro-fuzzy predictions. In Prognostics and Health Management Conference, 2010. PHM'10. (pp. 1-10). IEEE.

[38] El-Koujok, M., Gouriveau, R., \& Zerhouni, N. (2011). Reducing arbitrary choices in model building for prognostics: An approach by applying parsimony principle on an evolving neuro-fuzzy system. Microelectronics reliability, 51(2), 310-0.

[39] Javed, K., Gouriveau, R., \& Zerhouni, N. (2013, November). Novel failure prognostics approach with dynamic thresholds for machine degradation. InIndustrial Electronics Society, IECON 2013-39th Annual Conference of the IEEE. 
[40] Gouriveau, R., \& Zerhouni, N. (2012). Connexionist-systems-based long term prediction approaches for prognostics. Reliability, IEEE Transactions on, 61(4), 909-920.

[41] Li, X., Qian, J., \& Wang, G. G. (2013). Fault prognostic based on hybrid method of state judgment and regression. Advances in Mechanical Engineering, 2013.

[42] Ishibashi, R., \& Lucio Nascimento Junior, C. (2013, June). GFRBS-PHM: A Genetic Fuzzy Rule-Based System for PHM with Improved Interpretability. InPrognostics and Health Management (PHM), 2013 IEEE Conference on (pp. 1-7). IEEE.

[43] Jianzhong, S., Hongfu, Z., Haibin, Y., \& Pecht, M. (2010, January). Study of ensemble learning-based fusion prognostics. In Prognostics and Health Management Conference, 2010. PHM'10. (pp. 1-7). IEEE.

[44] Moghaddass, R., \& Zuo, M. J. (2014). An integrated framework for online diagnostic and prognostic health monitoring using a multistate deterioration process. Reliability Engineering \& System Safety, 1, 92-104.

[45] Xu, J., Wang, Y., \& Xu, L. (2014). PHM-oriented integrated fusion prognostics for aircraft engines based on sensor data. IEEE Sensors Journal, 14(4), 1124-1132.

[46] Wang, T., Yu, J., Siegel, D., \& Lee, J. (2008, October). A similarity-based prognostics approach for remaining useful life estimation of engineered systems. In Prognostics and Health Management, 2008. PHM 2008. International Conference on (pp. 1-6). IEEE.

[47] Ye, Z. S., Chen, N., \& Shen, Y. (2015). A new class of Wiener process models for degradation analysis. Reliability Engineering \& System Safety, 139, 58-67.

[48] Chen, N., Ye, Z. S., Xiang, Y., \& Zhang, L. (2015). Condition-based maintenance using the inverse Gaussian degradation model. European Journal of Operational Research, 243(1), 190-199.

[49] Ye, Z. S., \& Chen, N. (2014). The inverse Gaussian process as a degradation model. Technometrics, 56(3), 302-311. 
[50] Zhang, Y., \& Liao, H. (2015). Analysis of destructive degradation tests for a product with random degradation initiation time. Reliability, IEEE Transactions on, 64(1), 516-527.

[51] Shu, Y., Feng, Q., \& Coit, D. W. (2015). Life distribution analysis based on Lévy subordinators for degradation with random jumps. Naval Research Logistics, 62(6), 483-492.

[52] Liu, K., \& Huang, S. (2014). "Integration of data fusion methodology and degradation modeling process to improve prognostics." IEEE Transactions on Automation Science and Engineering, 13(1), 344-354.

[53] Liu, K., Chehade, A., \& Song, C, (2015). "Optimize the Signal Quality of the Composite Health Index Via Data Fusion for Degradation Modeling and Prognostic Analysis." IEEE Transactions on Automation Science and Engineering, 99(3), 1-11. 